\title{
Contactless Monitoring of Microcirculation Reaction on Local Temperature Changes
}

\author{
Maxim A. Volynsky ${ }^{1}$, Nikita B. Margaryants ${ }^{1}$, Oleg V. Mamontov ${ }^{1,2,3}$ and \\ Alexei A. Kamshilin 1,*(D) \\ 1 Faculty of Applied Optics, ITMO University, 49 Kronverksky ave., Saint Petersburg 197101, Russia; \\ maxim.volynsky@gmail.com (M.A.V.); fosp@grv.ifmo.ru (N.B.M.); mamontoffoleg@gmail.com (O.V.M.) \\ 2 Faculty of Medicine, Pavlov First Saint Petersburg State Medical University, 6-8 L'va Tolstogo str., Saint \\ Petersburg 197022, Russia \\ 3 Department of Circulation Physiology, Almazov National Medical Research Centre, 2 Akkuratova str., \\ Saint Petersburg 197341, Russia \\ * Correspondence: alexei.kamshilin@yandex.ru
}

Received: 18 October 2019; Accepted: 14 November 2019; Published: 17 November 2019

Featured Application: The study of blood vessel reactivity to local thermal impact could be used for assessment of the endothelial function in patients with increased cardio-metabolic risk (hypertension disease, diabetes mellitus, dyslipidemia). Moreover, it can be applied for revealing the subclinical impairments of blood flow in patients with rheumatologic pathology. Imaging photoplethysmography is a basis for the development of medical equipment capable of contactless assessment of the endothelial function in various skin regions.

\begin{abstract}
Assessment of skin blood flow is an important clinical task which is required to study mechanisms of microcirculation regulation including thermoregulation. Contactless assessment of vasomotor reactivity in response to thermal exposure is currently not available. The aim of this study is to show the applicability of the imaging photoplethysmography (IPPG) method to measure quantitatively the vasomotor response to local thermal exposure. Seventeen healthy subjects aged $23 \pm 7$ years participated in the study. A warm transparent compress applied to subject's forehead served as a thermal impact. A custom-made IPPG system operating at green polarized light was used to monitor the subject's face continuously and simultaneously with skin temperature and electrocardiogram (ECG) recordings. We found that the thermal impact leads to an increase in the amplitude of blood pulsations (BPA) simultaneously with the skin temperature increase. However, a multiple increase in BPA remained after the compress was removed, whereas the skin temperature returned to the baseline. Moreover, the BPA increase and duration of the vasomotor response was associated with the degree of external heating. Therefore, the IPPG method allows us to quantify the parameters of capillary blood flow during local thermal exposure to the skin. This proposed technique of assessing the thermal reactivity of microcirculation can be applied for both clinical use and for biomedical research.
\end{abstract}

Keywords: imaging photoplethysmography; blood microcirculation; thermoregulation

\section{Introduction}

Skin blood flow plays an important role in thermoregulation processes of the human body as a whole [1,2], allowing for the maintenance of a constant core temperature in spite of changes in the temperature of either body or environment. Notably, the thermoreceptors in the skin respond to temperature as well as to the rate of change of temperature [3]. The complex of thermoregulatory processes in the body provide thermal comfort [4]. 
Despite a large number of methods for assessing thermal sensation, an active search is underway for new methods that could clarify the mechanisms of thermoregulation [3]. The importance of considering vascular reactivity in response to temperature effects has been confirmed in a number of clinical studies, described in detail in respective reviews. Thus, the importance of a cold vasoconstrictor test (the implementation of which is closely related to the state of endothelial function and activation of the autonomic nervous system) for the prognosis of patients with cardiovascular diseases is underlined in reference [5]. Raynaud's syndrome, which develops in various rheumatological and vascular diseases, presents a separate problem. It is manifested by the increased reactivity of microvessels in response to cold stress. However, preclinical assessment of vascular reactivity is possible only with the help of the nailfold capillaroscopy [6]. Diseases such as type 2 diabetes mellitus (DM) or Raynaud's disease cause a disturbance of blood microcirculation or local neural activity, which leads to impaired thermoregulation and thermal sensation, both locally (mainly in the limbs) and generally, thus increasing the risk of heat exhaustion or heat stroke [7]. The relationship between the reaction of microvessels in response to heating and the European scale of global risk SCORE in patients with hypertension was demonstrated in [8]. The study of microvascular reactivity to temperature changes is even more clinically important in diabetic patients [9].

Therefore, the temperature reactivity of microvasculature is an actual pathophysiological component of many processes, but its study in a clinical environment is limited by the lack of reliable hardware assessment methods. The reaction of the microvasculature to temperature effects is often assessed by using indirect methods, such as measuring the dynamics of blood pressure or neurohormones. Despite the variety of non-invasive methods to investigate the skin microcirculation including capillaroscopy, optical coherence tomography, laser Doppler flowmetry (LDF), photoplethysmography (PPG), thermography and near-infrared spectroscopy [10], only a few of them have been used to assess the temperature reactivity, and experiments have usually been carried out in laboratory conditions. Particularly, it was shown that changes in skin blood flow measured by LDF and PPG are related to changes in the skin temperature [11]. Recently, the possibility of quantifying the change in blood microcirculation with local temperature effects on the human hand has been demonstrated by using the imaging photoplethysmography (IPPG) system [12]. It is worth noting that the PPG waveform was measured by Kamshilin et al. [12] only afterwards the thermal impact. However, monitoring of the processes before, during, and after temperature changes would provide more information about the vascular processes. In addition, most studies of the relationship between local skin temperature and microcirculation have been carried out on the subjects' fingers. It is well known that the temperature impact on different bodys' regions leads to different reactions of the circulatory system. For example, immersing one's hand in cold water or applying ice to the chest can cause systemic vasoconstriction and tachycardia, whereas cooling the face is often accompanied by bradycardia. Differences in the local regulation of microvascular tone are also possible. Therefore, contactless measurements of PPG waveform parameters in the facial area during heat exposure can differ significantly from local reactions in the upper and lower extremities. In addition, different regions may be involved in the pathological process of different diseases.

In this paper, we carried out the first pilot study showing the possibility of quantitatively assessing variations in skin temperature and cutaneous blood flow caused by an external, local heat compress in the facial area using the IPPG method. It was found that in response to a slight warming of the forehead $\left(2-7^{\circ} \mathrm{C}\right)$, skin blood flow initially increases exclusively in this area, and after compress removal, the increased microcirculation remains despite the skin temperature diminishing.

\section{Materials and Methods}

Figure 1A shows the layout of the experimental setup consisted of an illumination module and a data recording system. The illumination module (Figure 1B) included eight light-emitting diodes (LEDs) operating at a wavelength of $525 \mathrm{~nm}$ with a power of $3 \mathrm{~W}$ for each LED. The data recording system contained three detectors. First, a digital monochrome CMOS camera (GigE uEye UI-5220SE of 
the Imaging Development Systems GmbH, Obersulm, Germany) for recording a sequence of subject's face images with a size of $752 \times 480$ pixels at a frame rate of 39 frames per second.

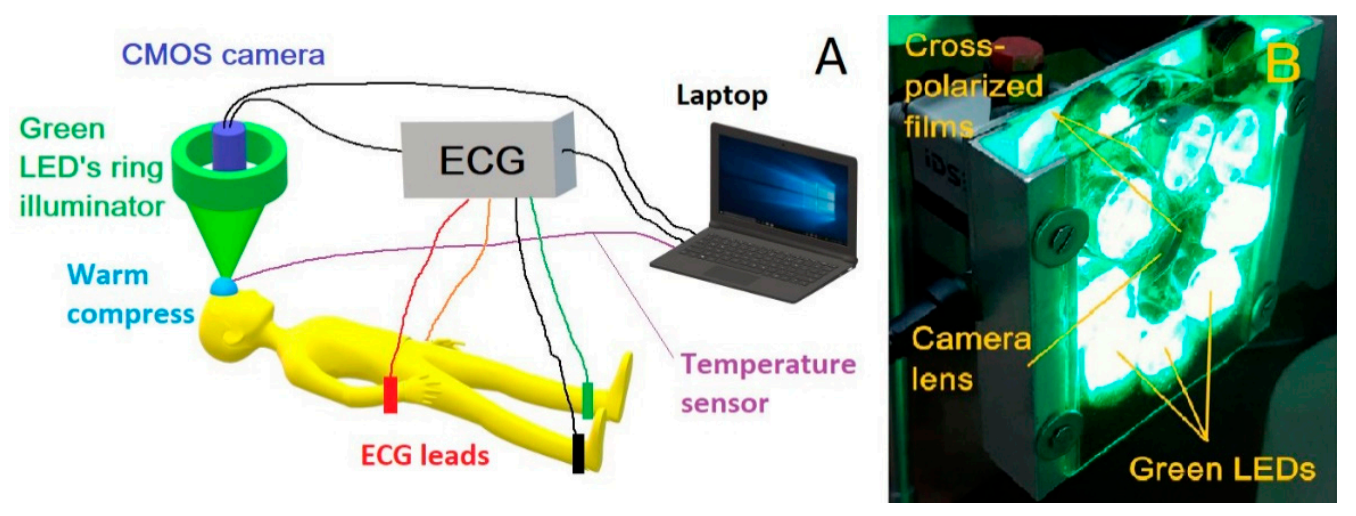

Figure 1. Schematic view of the experimental setup (A) and photograph of the illuminating module including video camera (B).

Second, a digital electrocardiograph (EKG) (KAP-01-“Kardiotekhnica-EKG” of the Incart Ltd., Saint Petersburg, Russia) for recording electrocardiograms (ECG) at the sampling rate of $1 \mathrm{kHz}$ in two leads. Third, an electronic USB-thermometer (RODOS-5 of the Olymp Ltd., Zelenograd, Moscow, Russia) with three semiconductor temperature sensors (DS18B20 of the Maxim Integrated, San Jose, CA, USA) for the monitoring of skin temperature in the forehead, cheek and laboratory room. The subjects' face illumination and recording of video data were done through cross-polarized polarization films to minimize motion artefacts [13]. Synchronization of the digital camera and ECG was provided with an accuracy of $1.0 \mathrm{~ms}$. Temperature data were recorded every $2 \mathrm{~s}$ synchronously with the recording of video frames. A detailed description of the experimental setup is given in our recent paper [14]. Video frames, ECG and temperature data were downloaded to the computer's hard drive for subsequent offline processing.

A distinctive feature of our imaging photoplethysmography (IPPG) system would be the video recordings of the subject's skin under green illumination. We used this illumination because the greatest light modulation resulting from the heart rate was observed at this wavelength $[15,16]$, despite its small penetration depth due to strong absorption by red blood cells [17]. Green light does not interact directly with pulsatile arteries and arterioles, but pulsatile arterial pressure mechanically deforms the upper layer of the capillary bed, thus modulating absorption and scattering coefficients of the light, according to the recently proposed model of PPG waveform formation [18]. Therefore, parameters of the microcirculation in the upper layer of the dermis were assessed in our experiments.

The experiment was conducted according to the following protocol. The subject was in the supine position while the video camera was mounted above his or her head. After $15 \mathrm{~min}$ of relaxation time for the subject, their baseline data (video, ECG and skin temperature) were recorded for about $5 \mathrm{~min}$. Then a warm compress was applied to their forehead. The compress was a 3 by $4 \mathrm{~cm}$ sealed bag made of transparent plastic, filled with water, and preheated in a thermostat to a temperature of 38-39 ${ }^{\circ} \mathrm{C}$. The compress was applied without interrupting the video recording, ECG and temperature. The heated compress was on the subject's forehead for about $6 \mathrm{~min}$, and then it was removed, again without interrupting the data recording. Thereafter, we recorded the whole set of data for more than $5 \mathrm{~min}$ to evaluate the relaxation processes. Air temperature in the laboratory room during the whole experiment was kept at the level of $23 \pm 1{ }^{\circ} \mathrm{C}$.

Recorded video, ECG and temperature data were processed by using custom software implemented in the MATLAB ${ }^{\circledR}$ platform. The algorithm of PPG signal processing was described in detail in our previous papers $[14,19]$. Briefly, we calculated the spatial distribution of the blood pulsation amplitude (BPA) over the facial area. To this end, in one of recorded images, we manually selected an area for analysis containing the forehead and cheeks. This area was divided into a network of small regions of 
interest (ROIs), each of the size of $5 \times 5$ pixels that covers an area of about $1.5 \mathrm{~mm} \times 1.5 \mathrm{~mm}$ on the face. BPA in every small ROI was estimated as

$$
\mathrm{BPA}=(1-\mathrm{AC} / \mathrm{DC}) \times 100 \%,
$$

where AC is the amplitude of an alternating component of the PPG signal, and DC is its slowly varying component. A typical example of a raw waveform calculated as the pixel value averaged within one of small ROIs is shown in Figure 2A by the blue curve. The brown line in this graph shows the DC component used to estimate the amplitude of blood pulsations (see Equation (1)). The black curve in Figure 2A shows our simultaneously-recorded ECG signal. R-peaks of the ECG signal were used to evaluate the mean shape of the pulse over 30 cardiac cycles, as illustrated in Figure 2B [14]. Thin colored lines in this graph show the PPG waveforms of each cardiac cycle plotted so that their beginning coincides with the respective R-peak. The thick green line is the mean waveform from which the BPA was estimated as the difference between its maximum and minimum. Depending on the heart rate, 30 cardiac cycles lasted from 22 to $35 \mathrm{~s}$ in our cohort of subjects defining the temporal resolution of BPA estimation. This algorithm allowed us to obtain BPA maps of the subject's face and measure their variations due to the warm compress application.
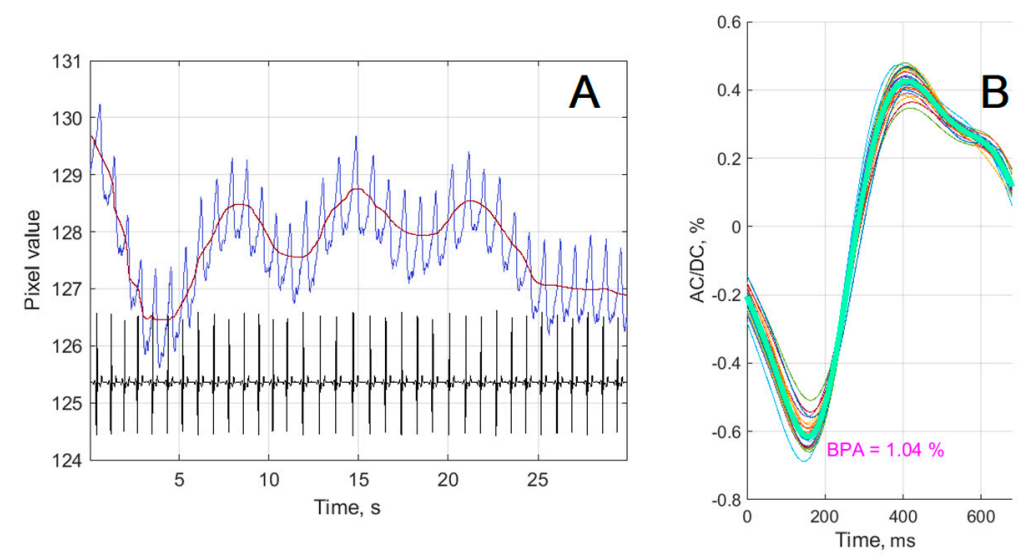

Figure 2. (A) An example of a raw photoplethysmography (PPG) waveform measured in a small region of interest (ROI) of $5 \times 5$ pixels (blue curve) along with a simultaneously-recorded electrocardiogram (ECG) (black curve). The brown line shows a slowly varying direct current (DC) component of the waveform. (B) Mean alternating current/direct current (AC/DC) ratio (green curve) after averaging of 30 successive cardiac cycles (thin colored lines).

Seventeen healthy and mostly young volunteers aged between 18-48 years (mean age of 23 years) were involved in this study (Table 1). This study was conducted in accordance with the ethical standards laid down in the 1964 Declaration of Helsinki, Guidelines for ethic committees conducting expertise of bio-medical studies of the World Health Organization (WHO) and European Forum for Good Clinical Practice (EF GCP). All experiments were approved by the local ethical committee of the Federal Almazov North-West Medical Research Centre (protocol of 08.02.2016, supervisor: M.D., Dr. Oleg V. Mamontov) prior to the experiments. All subjects gave their written, informed consent of participation in the experiment with permission to use their initials (without a full name), photographs of faces and data about their health in this article. 
Table 1. Subjects who participated in the study.

\begin{tabular}{|c|c|c|c|c|c|c|}
\hline Subject & Age, Years & Gender & $\begin{array}{l}\text { Height, } \\
\text { cm }\end{array}$ & Weight, kg & $\begin{array}{c}\text { Arterial Blood } \\
\text { Pressure, } \mathrm{mmHg}\end{array}$ & Pulse, bpm \\
\hline ZDV & 20 & M & 171 & 67 & $123 / 77$ & 82 \\
\hline BVE & 22 & M & 184 & 75 & $138 / 86$ & 81 \\
\hline CMA & 23 & M & 170 & 65 & $132 / 79$ & 81 \\
\hline VMA & 31 & M & 175 & 75 & $130 / 90$ & 75 \\
\hline KVO & 19 & M & 164 & 48 & $97 / 68$ & 60 \\
\hline EIA & 19 & M & 175 & 65 & $107 / 52$ & 53 \\
\hline RDI & 19 & M & 165 & 74 & $132 / 75$ & 82 \\
\hline GRD & 20 & $\mathrm{M}$ & 168 & 93 & $121 / 79$ & 79 \\
\hline FDA & 18 & $\mathrm{~F}$ & 170 & 56 & $122 / 80$ & 67 \\
\hline KGA & 18 & M & 185 & 70 & $119 / 70$ & 67 \\
\hline SVS & 20 & $\mathrm{~F}$ & 170 & 60 & $113 / 76$ & 58 \\
\hline MNB & 48 & M & 175 & 90 & $124 / 88$ & 76 \\
\hline GLU & 24 & $\mathrm{~F}$ & 168 & 60 & $121 / 94$ & 70 \\
\hline KVS & 22 & M & 178 & 80 & $128 / 81$ & 73 \\
\hline AME & 20 & $\mathrm{M}$ & 185 & 75 & $125 / 78$ & 72 \\
\hline SAA & 19 & M & 180 & 71 & $135 / 72$ & 86 \\
\hline SKP & 22 & M & 178 & 80 & $128 / 81$ & 73 \\
\hline
\end{tabular}

\section{Results}

A set of two-dimensional BPA distributions in the selected area of the face of each subject was calculated every 21-34 s (depending on the subject's heart rate) during the entire measurement according to the protocol described above. Examples of such distributions overlaid with respective images of the subject's face are shown in Figure 3A-C. In this particular experiment, the first BPA map (Figure 3A) was calculated at the end of the first minute of the experiment (baseline), the second (Figure 3B) at the sixth minute (with the heated compress) and the third (Figure 3C) at the fifteenth minute when the compress was removed. The red color in the BPA maps shows the points with a higher amplitude of pulsations, while the blue color corresponds to the lower BPA. The color scale of BPA (common for all three maps) is shown on the right of Figure 3C. One can see that the amplitude of blood pulsations significantly increases in the forehead under the warm compress, whereas it remains unchanged in other areas. It is worth noting the difference in BPA maps before and 4 min after applying the compress (Figure 3A vs. Figure 3C); despite the absence of the compress, blood pulsations in the forehead remained doubled, whereas no changes were observed in other areas. 

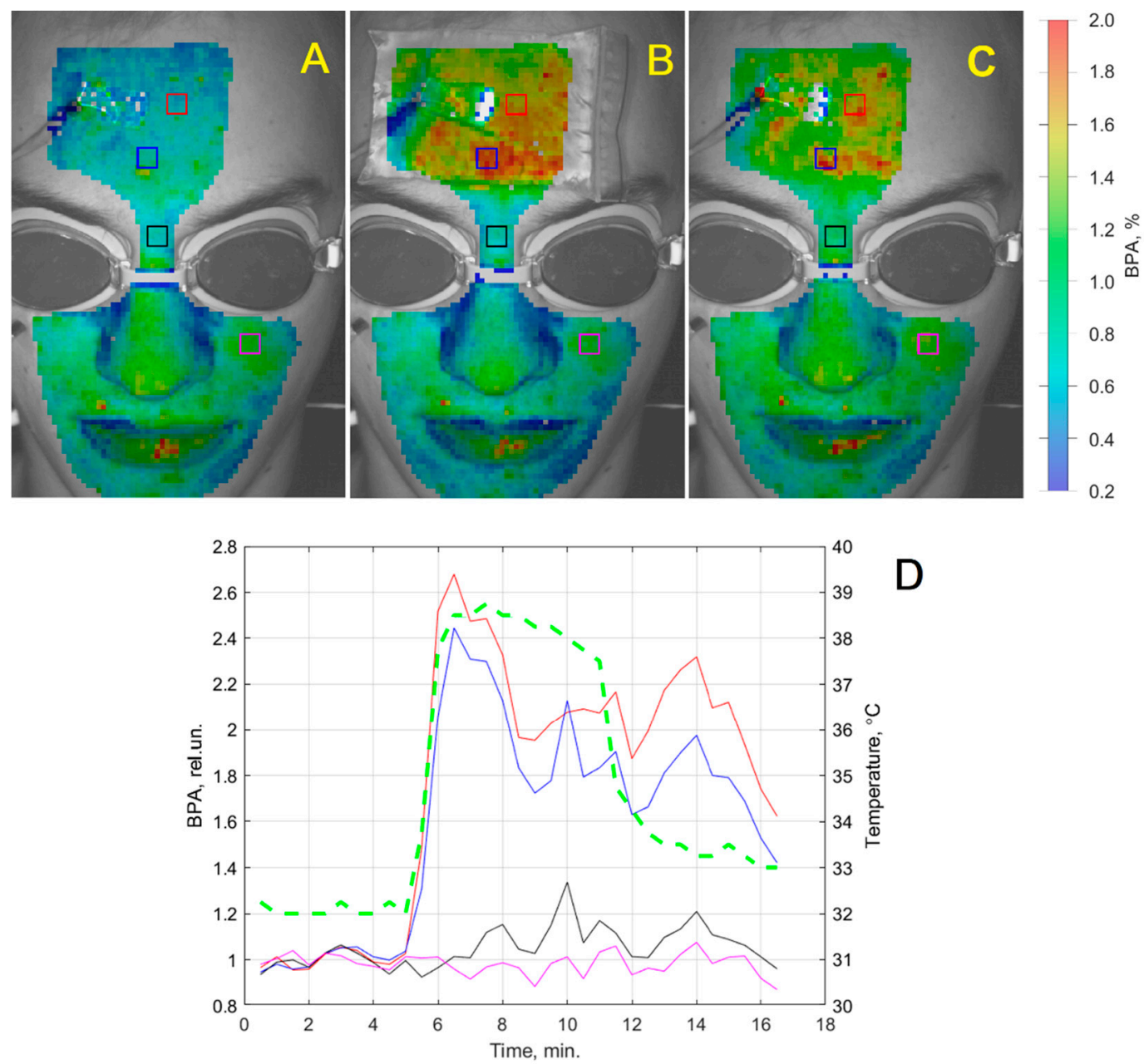

Figure 3. Spatial distribution of amplitude of blood pulsations (BPA) overlaid with an image of the subject's face before (A), during (B), and after (C) applying the warm compress. BPA maps are shown in pseudocolors with the common color scale in percent on the right of the panel (C). Panel (D) shows evolution of BPA (solid color lines with the scale on the left) along with skin temperature (dashed green line with the scale on the right). Red and blue lines show BPA averaged in the ROIs located at the forehead in the area of the compress application and marked by the red and blue squares in panels $(\mathrm{A}-\mathrm{C})$, respectively. Black and magenta lines in graph (D) show BPA dynamics estimated in the black and magenta ROIs located in the nose and cheek, respectively. The application of the compress lasted from the fifth to the eleventh minute of the experiment.

To monitor BPA changes in different facial areas, we chose four larger ROIs measuring $35 \times 35$ pixels in the image that corresponds to $7.5 \mathrm{~mm} \times 7.5 \mathrm{~mm}$ in the subject's face. Two of them were chosen in the forehead in the middle of the compress application, and two others (reference regions) in the nose and on the cheek. These ROIs are shown in Figure 3A-C by squares of different colors: red and blue in the forehead, black on the bridge of the nose and magenta on the cheek. The evolution of the BPA averaged within four large ROIs is shown in Figure 3D. The color of the curves corresponds to the color of the ROIs in Figure 3A-C. The dashed green line in this graph shows the dynamics of the skin temperature measured in the area of the compress application. For a particular subject shown in Figure 3, the compress was applied on the fifth minute of the experiment and removed on the eleventh one.

It is seen that BPA increases sharply after applying the compress, and then it diminishes with the tendency to reach a steady state. Typically, it remains at a level significantly higher than the baseline 
during the whole period of the thermal impact, and in the most cases, it continues at this level even after the termination of the impact.

The observed BPA increase reflects the vasomotor response of the skin to a local temperature increase, the physiological equivalent of which is an increase in the number of functioning capillaries. Therefore, most likely, BPA is a measure of the intensity of blood flow in the upper layers of the skin.

As seen in Figure 3, the amplitude of the PPG waveform in the skin exposed to the thermal impact (red and blue curves in Figure 3D) displays different dynamics than that in the non-exposed areas (black and magenta curves). While a significant increase in BPA was observed in the compress application area, no such dynamics was observed in the reference ROIs. A significant BPA difference between the heated and unheated facial areas before and during compress application was observed for all subjects $(p<0.0001)$, as shown in Figure 4. Hereinafter, a paired-sample $t$-test is used to evaluate the statistical significance of the difference between these data groups.

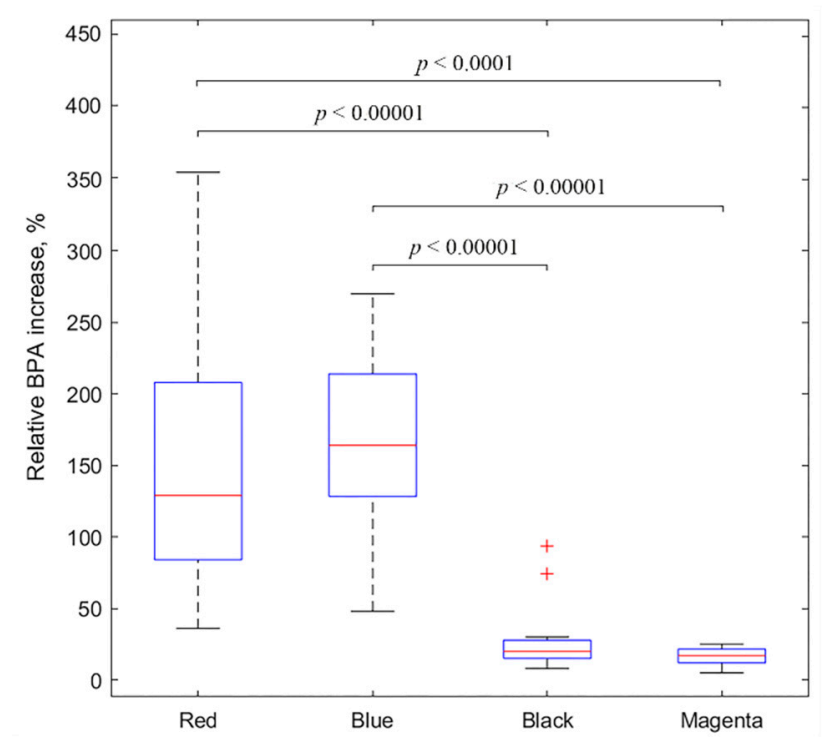

Figure 4. Vasomotor response to the thermal impact applied to the forehead, measured as the relative increase of BPA in different locations before and during compress application, and presented as a "box-and-whiskers" type diagram for all studied subjects. Red and blue are ROIs located on the forehead, black is ROI located in the bridge of the nose and magenta in the cheek.

The intensity of the thermal impact denoted as heating grade and measured as the difference in the skin temperature in the forehead before and after application of the compress was different for different subjects (see Table 2). Periods of the compress application denoted as heating time were also slightly different. The duration of the vasomotor reaction of skin vessels to the heat exposure was determined as a period during which BPA exceeds its mean level plus the standard deviation (STD) measured in the baseline. The mean heating time was $6.2 \pm 1.5 \mathrm{~min}$ whereas the mean duration of the vasomotor reaction in the entire cohort of subjects was $8.9 \pm 2.6 \mathrm{~min}$. The example of BPA dynamics in Figure 3D shows that its initial growth occurs immediately with increasing temperature and takes the same time (30-40 s). A similar reaction was observed in all studied subjects. After removing the compress, BPA in the forehead remained significantly higher than in the baseline in 10 of 17 subjects, despite the fact that the skin temperature of the forehead always dropped to the initial level. We explain this observation by differences in the heating grade, which was different in different experiments. A simultaneous drop in BPA and skin temperature was observed in subjects for whom the heating grade was less than $3.5^{\circ} \mathrm{C}$ (with the exception for KVO), as seen in Table 2. More pronounced heating of the skin was accompanied by a longer reaction of the vascular system.

Table 2 also shows the mean BPA $( \pm$ STD) in the forehead before, during and after the thermal impact for each subject. As one can see, there is a significant increase in the parameter BPA for all 
measured subjects during the warm compress application. Correlation analysis of the data has revealed that both mean BPA after the impact and its absolute increase in respect to the baseline positively correlate with the heating grade: $r=0.73, p=0.001$ and $r=0.77, p<0.001$, respectively. Nevertheless, no correlation of the BPA increase with age, gender, blood pressure and the mass index was observed within our cohort of subjects.

Table 2. Heating grade, duration of vasomotor response, and mean BPA measured in the forehead before, during, and after the thermal impact in different subjects.

\begin{tabular}{ccccccc}
\hline Subject & $\begin{array}{c}\text { Heating } \\
\text { Grade, }{ }^{\circ} \mathbf{C}\end{array}$ & $\begin{array}{c}\text { Heating } \\
\text { Time, min. }\end{array}$ & $\begin{array}{c}\text { Response } \\
\text { Duration, } \\
\text { min. }\end{array}$ & $\begin{array}{c}\text { Mean BPA } \\
\text { before } \\
\text { Impact, \% }\end{array}$ & $\begin{array}{c}\text { Mean BPA } \\
\text { during } \\
\text { Impact, \% }\end{array}$ & $\begin{array}{c}\text { Mean BPA } \\
\text { after Impact, } \\
\text { \% }\end{array}$ \\
\hline ZDV & 1.0 & 7.0 & 7.0 & $0.39 \pm 0.01$ & $0.58 \pm 0.08$ & $0.39 \pm 0.06$ \\
BVE & 1.9 & 6.0 & 6.5 & $0.55 \pm 0.07$ & $0.86 \pm 0.04$ & $0.53 \pm 0.02$ \\
CMA & 2.0 & 6.0 & 6.0 & $0.50 \pm 0.23$ & $0.85 \pm 0.23$ & $0.44 \pm 0.15$ \\
VMA & 2.4 & 6.5 & 6.5 & $0.57 \pm 0.04$ & $0.63 \pm 0.06$ & $0.62 \pm 0.03$ \\
KVO & 2.5 & 7.0 & 11.0 & $0.48 \pm 0.02$ & $0.68 \pm 0.08$ & $0.46 \pm 0.07$ \\
EIA & 3.0 & 6.0 & 7.0 & $0.69 \pm 0.07$ & $1.35 \pm 0.32$ & $0.84 \pm 0.10$ \\
RDI & 3.3 & 6.0 & 6.5 & $0.56 \pm 0.14$ & $1.02 \pm 0.29$ & $0.66 \pm 0.23$ \\
GRD & 3.5 & 7.0 & 7.0 & $0.44 \pm 0.02$ & $0.56 \pm 0.09$ & $0.48 \pm 0.02$ \\
FDA & 4.2 & 7.5 & 11.5 & $0.44 \pm 0.02$ & $1.31 \pm 0.22$ & $1.14 \pm 0.10$ \\
KGA & 4.9 & 6.5 & 11.5 & $1.08 \pm 0.05$ & $2.24 \pm 0.61$ & $1.32 \pm 0.15$ \\
SVS & 5.0 & 6.0 & 11.0 & $0.46 \pm 0.02$ & $0.94 \pm 0.15$ & $0.85 \pm 0.06$ \\
MNB & 5.3 & 7.0 & 11.5 & $0.48 \pm 0.04$ & $0.71 \pm 0.06$ & $0.55 \pm 0.02$ \\
GLU & 6.1 & 6.0 & 11.0 & $0.51 \pm 0.04$ & $0.96 \pm 0.18$ & $0.73 \pm 0.18$ \\
KVS & 6.6 & 5.5 & 9.5 & $0.43 \pm 0.03$ & $1.13 \pm 0.15$ & $0.94 \pm 0.14$ \\
AME & 6.7 & 6.0 & 13.0 & $0.73 \pm 0.10$ & $1.58 \pm 0.19$ & $1.28 \pm 0.12$ \\
SAA & 7.6 & 6.5 & 11.0 & $0.87 \pm 0.09$ & $1.02 \pm 0.25$ & $1.09 \pm 0.18$ \\
SKP & 8.6 & 5.5 & 9.5 & $0.78 \pm 0.04$ & $1.36 \pm 0.22$ & $1.09 \pm 0.13$ \\
\hline
\end{tabular}

Note that the skin temperature decreased immediately after the compress removal with the typical relaxation time being less than a minute. However, BPA significantly exceeded the baseline even five minutes later, resulting in a significantly larger mean BPA $(p<0.05)$ after cessation of the thermal impact for subjects to whom a compress heating the forehead by more than $4{ }^{\circ} \mathrm{C}$ was applied (see the lower half of Table 2).

\section{Discussion and Conclusions}

Our pilot study has revealed that the contactless IPPG method can be used for assessing the hemodynamic changes caused by local thermal impact. We have found that BPA is a very sensitive marker of changes in skin blood flow that are observed in response to local heating of the forehead skin. A distinctive feature of our study (achieved due to use of a transparent warm compress) is the coincidence of the locally heated region with the area in which the parameters of PPG waveforms are assessed with high spatial resolution. This feature increases the reliability of the processed experimental data, since BPA reflects the state of microcirculation variation due to local thermal exposure, while simultaneously controlling blood flow in areas in which this exposure was not applied. Using the IPPG method, it is possible to select a localization region and compensate for the spatially heterogeneous distribution of blood vessels. This is due to subsequent averaging of the signal over the chosen area, with reference to the BPA parameter. In contrast, the LDF fiber probe, which is widely used for microcirculation assessment, cannot be applied for measurements through the compress. Moreover, it does not allow the assessment of microcirculation parameters simultaneously in the large facial area with high spatial resolution.

Our study has also revealed that an increase of the BPA parameter and duration of the vasomotor response, caused by a few degrees of local heating, vary substantially. However, several universal features were observed. (i) The vasomotor response is clearly distinguishable by the IPPG method and 
characterized by a several-fold increase of BPA. (ii) The response is localized in the area of thermal impact and arises immediately with impact.

(iii) In most cases, the increase in BPA continues after the cessation of heat exposure and the respective diminishing of the skin temperature. These features were observed in all healthy subjects who participated in the experiment. The latest observation shows that it is the local temperature impact that leads to changes in BPA. These changes were not caused by additional light reflections from the pair of plastic-water interfaces introduced by the compress. Despite that the plastic bag with water affects the transmitted light, the AC/DC ratio used for evaluation of BPA in our algorithm (see Equation (1)) is almost independent from light intensity variations. Figure $3 \mathrm{~B}$ shows that the features of the spatial distribution of BPA assessed through the plastic bag are similar (but with higher amplitude) as in Figure $3 \mathrm{C}$ after removing the compress. We suggest that the proposed heating test and its monitoring have important diagnostic value for local regulation of microcirculation in patients having different diseases of pathogenesis characterized by factors violating the neurogenic and endothelial regulation of microcirculation [20,21].

It should be noted that our previous experiments, in which applicability of an IPPG system to assess the microvasculature response on the temperature changes [12] was demonstrated, were carried out in conditions under which the temperature of a preliminarily-cooled finger was increased due to internal heating. In contrast, here we assessed the microcirculation reaction to the external local thermal impact with the temperature exceeding the skin temperature. Our near-future plans include carrying out a study to monitor the microcirculation reaction to the application of a cold compress (below the forehead temperature).

It is worth noting that the proposed technique allows measuring the vascular response to the local heat exposure in almost any area of human skin. This is also a valuable feature, since in many diseases the changes in microcirculation are local in nature, which makes it necessary to carry out diagnostic studies in specific regions. The observed correlation between the BPA increase and the intensity of the thermal impact could be explained by previous observations demonstrating that a larger number of different TRP channels are involved in the vascular reaction to the temperature increase [22]. The response of each type of channel is likely summarized with the increasing temperature of the impact. Therefore, the revealed differences in the hemodynamic response with the different intensity of the thermal exposure create the prerequisite for the differential assessment of the function of various types of TRP channels involved in thermoregulation.

In conclusion, the method for assessing local microcirculation demonstrated in this work has the potential to be a valuable diagnostic tool that allows for the first time to reveal the direct response of microvessels to the temperature exposure at the place of its application. To realize this potential successfully, it is necessary to carry out further studies of the vasomotor response in various regions and under different temperature exposure in patients with diseases such as diabetes mellitus, systemic scleroderma, obliterating vascular diseases, as well as in patients with cardiovascular risk factors.

Author Contributions: Conceptualization, A.A.K., O.V.M.; methodology, A.A.K., O.V.M.; experimental setup design and manufacturing, A.A.K., M.A.V., N.B.M.; software design, A.A.K.; formal analysis, M.A.V., N.B.M.; investigation, M.A.V., N.B.M., A.A.K.; writing-original draft preparation, M.A.V., N.B.M., O.V.M.; writing-review and editing, A.A.K.; visualization, M.A.V.; supervision, A.A.K.

Funding: This research was funded by the Russian Science Foundation (grant number 15-15-20012) in terms of developing a conception and methodology of research, software development, experimental data collecting and processing. The Ministry of Science and Higher Education of the Russian Federation (project number 8.2501.2017/4.6) supported this research in terms of modifications of the experimental setup, including temperature control equipment and operational modes optimization for the optical-electronic part of the setup.

Conflicts of Interest: The authors declare no conflict of interest. 


\section{References}

1. Arens, E.A.; Zhang, H. The skin's role in human thermoregulation and comfort. In Thermal and Moisture Transport in Fibrous Materials.; Pan, N., Gibson, P., Eds.; Woodhead Publishing Ltd.: Sawston, UK, 2006; pp. 560-602.

2. Romanovsky, A.A. Skin temperature: its role in thermoregulation. Acta Physiol. 2014, 210, 498-507. [CrossRef] [PubMed]

3. Nagashima, K.; Tokizava, K.; Marui, S. Thermal comfort. In Thermoregulation: From Basic Neuroscience to Clinical Neurology Part I; Romanovsky, A.A., Ed.; Handbook of Clinical Neurology; Elsevier: Amsterdam, Netherlands, 2018; pp. 249-260, ISBN 978-0-444-63912-7.

4. Djongyang, N.; Tchinda, R.; Njomo, D. Thermal comfort: A review paper. Renew. Sustain. Energy Rev. 2010, 14, 2626-2640. [CrossRef]

5. Pouwels, S.; Van Genderen, M.E.; Kreeftenberg, H.G.; Ribeiro, R.; Parmar, C.; Topal, B.; Celik, A.; Ugale, S. Utility of the cold pressor test to predict future cardiovascular events. Expert Rev. Cardiovasc. Ther. 2019, 17, 305-318. [CrossRef] [PubMed]

6. Latuskiewicz-Potemska, J.; Chmura-Skirlinska, A.; Gurbiel, R.J.; Smolewska, E. Nailfold capillaroscopy assessment of microcirculation abnormalities and endothelial dysfunction in children with primary or secondary Raynaud syndrome. Clin. Rheumatol. 2016, 35, 1993-2001. [CrossRef] [PubMed]

7. Charkoudian, N. Skin blood flow in adult human thermoregulation: how it works, when it does not, and why. Mayo Clin. Proc. 2003, 78, 603-612. [CrossRef] [PubMed]

8. Jekell, A.; Kalani, M.; Kahan, T. The interrelation of endothelial function and microvascular reactivity in different vascular beds, and risk assessment in hypertension: results from the Doxazosin-ramipril study. Heart Vessels 2019, 34, 484-495. [CrossRef] [PubMed]

9. Östlund Papadogeorgos, N.; Jörneskog, G.; Bengtsson, M.; Kahan, T.; Kalani, M. Severely impaired microvascular reactivity in diabetic patients with an acute coronary syndrome. Cardiovasc. Diabetol. 2016, 15, 66. [CrossRef] [PubMed]

10. Wright, C.I.; Kroner, C.I.; Draijer, R. Non-invasive methods and stimuli for evaluating the skin's microcirculation. J. Pharmacol. Toxicol. Methods 2006, 54, 1-25. [CrossRef] [PubMed]

11. Allen, J.; Frame, J.R.; Murray, A. Microvascular blood flow and skin temperature changes in the fingers following a deep inspiratory gasp. Physiol. Meas. 2002, 23, 365-373. [CrossRef] [PubMed]

12. Kamshilin, A.A.; Belaventseva, A.V.; Romashko, R.V.; Kulchin, Y.N.; Mamontov, O.V. Local thermal impact on microcirculation assessed by imaging photoplethysmography. Biol. Med. 2016, 8, 1000361. [CrossRef]

13. Sidorov, I.S.; Volynsky, M.A.; Kamshilin, A.A. Influence of polarization filtration on the information readout from pulsating blood vessels. Biomed. Opt. Express 2016, 7, 2469-2474. [CrossRef] [PubMed]

14. Kamshilin, A.A.; Krasnikova, T.V.; Volynsky, M.A.; Miridonov, S.V.; Mamontov, O.V. Alterations of blood pulsations parameters in carotid basin due to body position change. Sci. Rep. 2018, 8, 13663. [CrossRef] [PubMed]

15. Fallow, B.A.; Tarumi, T.; Tanaka, H. Influence of skin type and wavelength on light wave reflectance. J. Clin. Monit. Comput. 2013, 27, 313-317. [CrossRef] [PubMed]

16. Kamshilin, A.A.; Margaryants, N.B. Origin of Photoplethysmographic Waveform at Green Light. Phys. Procedia 2017, 86, 72-80. [CrossRef]

17. Anderson, R.R.; Parrish, J.A. The optics of human skin. J. Invest. Dermatol. 1981, 77, 13-19. [CrossRef] [PubMed]

18. Kamshilin, A.A.; Nippolainen, E.; Sidorov, I.S.; Vasilev, P.V.; Erofeev, N.P.; Podolian, N.P.; Romashko, R.V. A new look at the essence of the imaging photoplethysmography. Sci. Rep. 2015, 5, 10494. [CrossRef] [PubMed]

19. Kamshilin, A.A.; Sidorov, I.S.; Babayan, L.; Volynsky, M.A.; Giniatullin, R.; Mamontov, O.V. Accurate measurement of the pulse wave delay with imaging photoplethysmography. Biomed. Opt. Express 2016, 7, 5138-5147. [CrossRef] [PubMed]

20. Strain, W.; Chaturvedi, N.; Hughes, A.; Nihoyannopoulus, P.; Bulpitt, C.; Rajkumar, C.; Shore, A.C. Associations between cardiac target organ damage and microvascular dysfunction: the role of blood pressure. J. Hypertens. 2010, 28, 952-958. [CrossRef] [PubMed] 
21. Strain, W.; Paldanius, P.M. Diabetes, cardiovascular disease and the microcirculation. Cardiovasc. Diabetol. 2018, 17, 57. [CrossRef] [PubMed]

22. Huang, J.; Zhang, X.; McNaughton, P.A. Modulation of temperature-sensitive TRP channels. Semin. Cell Dev. Biol. 2006, 17, 638-645. [CrossRef] [PubMed]

(C) 2019 by the authors. Licensee MDPI, Basel, Switzerland. This article is an open access article distributed under the terms and conditions of the Creative Commons Attribution (CC BY) license (http://creativecommons.org/licenses/by/4.0/). 